\title{
Definition of Instantons in Noncommutative Gauge Theory in Higher Dimensions
}

\author{
Harald Markum* \\ Atominstitut, Vienna University of Technology, Austria \\ E-mail: markumetuwien.ac.at

\section{Arifa Ali Khan} \\ Faculty of Applied Sciences, University of Taiz, Yemen \\ E-mail: arifa.ali-khan@physik.uni-regensburg.de
}

\begin{abstract}
Theories with noncommutative space-time coordinates represent alternative candidates of grand unified theories. We discuss $\mathrm{U}(1)$ gauge theory in 2 and 4 dimensions on a lattice with $\mathrm{N}$ sites. The mapping to a $\mathrm{U}(\mathrm{N})$ plaquette model in the sense of Eguchi and Kawai can be used for computer simulations. In 2D it turns out that the formulation of the topological charge leads to the imaginary part of the plaquette. Concerning 4D, the definition of instantons seems straightforward. We show that one can transcribe the plaquette and hypercube formulation to the matrix theory. It will be interesting to measure the topological charge on a noncommutative hypercube.
\end{abstract}

The XXVIII International Symposium on Lattice Field Theory

June 14-19, 2010

Villasimius, Sardinia, Italy

* Speaker. 


\section{Motivation}

In noncommutative geometry, where the coordinate operators $\hat{x}_{\mu}$ satisfy the commutation relation $\left[\hat{x}_{\mu}, \hat{x}_{v}\right]=i \theta_{\mu v}$, a mixing between ultraviolet and infrared degrees of freedom takes place [1]. So lattice simulations are a promising tool to get deeper insight into noncommutative quantum field theories. For noncommutative U(1) gauge there exists an equivalent matrix model which makes numerical calculations feasible [2].

The main topic of the underlying contribution is to discuss the topological charge of noncommutative $\mathrm{U}(1)$ gauge theory in two and in higher dimensions. In two dimensions the instanton configurations carry a topological charge $q$ which was shown being non-integer [3, 4]. We work out the definition of instantons in four and higher dimensions.

\section{Topology and Instantons in QCD}

The Lagragian of pure gluodynamics (the Yang-Mills theory with no matter fields) in Euclidean spacetime can be written as

$$
\mathscr{L}=\frac{1}{4 g^{2}} G_{\mu \nu}^{a} G_{\mu \nu}^{a}
$$

where $G_{\mu \nu}^{a}$ is the gluon field strength tensor

$$
G_{\mu \nu}^{a}=\partial_{\mu} A_{v}^{a}-\partial_{v} A_{\mu}^{a}+f^{a b s} A_{\mu}^{b} A_{v}^{c}
$$

and $f^{a b c}$ are structure constants of the gauge group considered. The classical action of the YangMills fields can be identically rewritten as

$$
S=\frac{1}{8 g^{2}} \int d x^{4}\left(G_{\mu \nu}^{a} \pm \tilde{G}_{\mu \nu}^{a}\right)^{2} \mp \frac{8 \pi^{2}}{g^{2}} Q
$$

where Q denotes the topological charge

$$
Q=\frac{1}{32 \pi^{2}} \int d x^{4} G_{\mu \nu}^{a} \tilde{G}_{\mu \nu}^{a}
$$

with

$$
\tilde{G}_{\mu \nu}^{a}=\frac{1}{2} \varepsilon_{\mu v \alpha \beta} G_{\alpha \beta}^{a}
$$

\section{Definition of the Topological Charge in Two Dimensions}

\subsection{Lattice Regularization of Noncommutative Two-Dimensional U(1) Gauge Theory}

The lattice regularized version of the theory can be defined by an analog of Wilson's plaquette action

$$
S=-\beta \sum_{x} \sum_{\mu<v} U_{\mu}(x) \star U_{v}(x+a \hat{\mu}) \star U_{\mu}(x+a \hat{v})^{\dagger} \star U_{v}(x)^{\dagger}+\text { c.c. }
$$

where the symbol $\hat{\mu}$ represents a unit vector in the $\mu$-direction and we have introduced the lattice spacing $a$. The link variables $U_{\mu}(x)$ are complex fields on the lattice satisfying the star-unitarity 
condition. The star-product [1] on the lattice can be obtained by rewriting its definition within noncommutatiuve derivatives in terms of Fourier modes and restricting the momenta to the Brillouin zone.

Let us define the topological charge for a gauge field configuration on the discretized twodimensional torus. In the language of fields, we define the topological charge as

$$
q=\frac{1}{4 \pi i} \sum_{x} \sum_{\mu \nu} \varepsilon_{\mu \nu} U_{\mu}(x) \star U_{v}(x+a \hat{\mu}) \star U_{\mu}(x+a \hat{v})^{\dagger} \star U_{v}(x)^{\dagger}
$$

which reduces to the usual definition of the topological charge in $2 \mathrm{~d}$ gauge theory

$$
q=\frac{1}{4 \pi} \int d^{2} x \varepsilon_{\mu v} G_{\mu v}
$$

in the continuum limit.

\subsection{Matrix-Model Formulation}

It is much more convenient for computer simulations to use an equivalent formulation, in which one maps functions on a noncommutative space to operators so that the star-product becomes nothing but the usual operator product, which is noncommutative. The action (3.1) can then be written as

$$
\begin{aligned}
S & =-N \beta \sum_{\mu \neq v} \operatorname{tr}\left\{\hat{U}_{\mu}\left(\Gamma_{\mu} \hat{U}_{v} \Gamma_{\mu}^{\dagger}\right)\left(\Gamma_{v} \hat{U}_{\mu}^{\dagger} \Gamma_{v}^{\dagger}\right) \hat{U}_{v}^{\dagger}\right\}+2 \beta N^{2} \\
& =-N \beta \sum_{\mu \neq v} \mathscr{Z}_{v \mu} \operatorname{tr}\left(V_{\mu} V_{v} V_{\mu}^{\dagger} V_{v}^{\dagger}\right)+2 \beta N^{2}
\end{aligned}
$$

where $V_{\mu} \equiv \hat{U}_{\mu} \Gamma_{\mu}$ is a $\mathrm{U}(N)$ matrix and $N$ is the linear extent of the original lattice. An explicit representation of $\Gamma_{\mu}$ in the $d=2$ case shall be given in Sec. 3.3. This is the twisted Eguchi-Kawai (TEK) model [5], which appeared in history as a matrix model equivalent to the large $N$ gauge theory [6]. We have added the constant term $2 \beta N^{2}$ to what we would obtain from (3.1) in order to make the absolute minimum of the action zero.

By using the map between fields and matrices, the topological charge (3.2) can be represented in terms of matrices as

$$
\begin{aligned}
q & =\frac{1}{4 \pi i} N \sum_{\mu v} \varepsilon_{\mu v} \operatorname{tr}\left\{\hat{U}_{\mu}\left(\Gamma_{\mu} \hat{U}_{v} \Gamma_{\mu}^{\dagger}\right)\left(\Gamma_{v} \hat{U}_{\mu}^{\dagger} \Gamma_{v}^{\dagger}\right) \hat{U}_{v}^{\dagger}\right\} \\
& =\frac{1}{4 \pi i} N \sum_{\mu v} \varepsilon_{\mu v} \mathscr{Z}_{v \mu} \operatorname{tr}\left(V_{\mu} V_{v} V_{\mu}^{\dagger} V_{v}^{\dagger}\right)
\end{aligned}
$$

\subsection{Classical Solutions}

The classical equation of motion was worked out in the literature [7, 3] for the action (3.4)

$$
V_{\mu}^{\dagger}\left(W-W^{\dagger}\right) V_{\mu}=W-W^{\dagger}
$$

with the unitary matrix $W$

$$
W=\mathscr{Z}_{v \mu} V_{\mu} V_{v} V_{\mu}^{\dagger} V_{v}^{\dagger}
$$


General solutions to this equation can be written in a block-diagonal form [7]

$$
V_{\mu}=\left(\begin{array}{cccc}
\Gamma_{\mu}^{(1)} & & & \\
& \Gamma_{\mu}^{(2)} & & \\
& & \ddots & \\
& & & \Gamma_{\mu}^{(k)}
\end{array}\right)
$$

by an appropriate $\mathrm{SU}(N)$ transformation, where $\Gamma_{\mu}^{(j)}$ are $n_{j} \times n_{j}$ unitary matrices, $j=1, \ldots, k$, satisfying the 't Hooft-Weyl algebra

$$
\begin{aligned}
\Gamma_{\mu}^{(j)} \Gamma_{v}^{(j)} & =Z_{\mu v}^{(j)} \Gamma_{v}^{(j)} \Gamma_{\mu}^{(j)} \\
Z_{12}^{(j)} & =Z_{21}^{(j) *}=\exp \left(2 \pi i \frac{m_{j}}{n_{j}}\right) \\
m_{j} & =\frac{n_{j}+1}{2}
\end{aligned}
$$

An explicit representation is given by the clock and shift operators, $Q$ and $P$

$$
\Gamma_{1}^{(j)}=P_{n_{j}}, \quad \Gamma_{2}^{(j)}=\left(Q_{n_{j}}\right)^{m_{j}}
$$

The action and the topological charge can then be evaluated as $[3,7]$

$$
\begin{aligned}
& S=4 N \beta \sum_{j} n_{j} \sin ^{2}\left\{\pi\left(\frac{m_{j}}{n_{j}}-\frac{M}{N}\right)\right\} \\
& q=\frac{N}{2 \pi} \sum_{j} n_{j} \sin \left\{2 \pi\left(\frac{m_{j}}{n_{j}}-\frac{M}{N}\right)\right\}
\end{aligned}
$$

In general, the topological charge $q$ is not an integer. If we require the action to be less than of order $N$ the argument of the sine has to vanish for all $j$. In that case the topological charge approaches an integer

$$
q \simeq N\left(\sum_{j} m_{j}-M\right)
$$

being a multiple of $N$.

\section{Definition of the Topological Charge in Four and Higher Dimensions}

The lattice action (3.1) taking into account the star-product can be used in any dimension. The field-theoretic definition of the topological charge (3.2) can be extended in two ways.

One can rely on the so-called plaquette definition which then yields a product of two plaquettes

$$
\begin{array}{r}
q^{(P)}=\frac{-1}{32 \pi^{2}} \sum_{x} \sum_{\mu v \rho \sigma} \varepsilon_{\mu v \rho \sigma} U_{\mu}(x) \star U_{v}(x+a \hat{\mu}) \star U_{\mu}(x+a \hat{v})^{\dagger} \star U_{v}(x)^{\dagger} \\
\star U_{\rho}(x) \star U_{\sigma}(x+a \hat{\rho}) \star U_{\rho}(x+a \hat{\sigma})^{\dagger} \star U_{\sigma}(x)^{\dagger}
\end{array}
$$

which reduces to the definition of the topological charge in $4 \mathrm{~d}$ gauge theory

$$
q^{(P)}=\frac{1}{32 \pi^{2}} \int d^{4} x \varepsilon_{\mu v \rho \sigma} G_{\mu v} \star G_{\rho \sigma}
$$


in the continuum limit.

By using the map between fields and matrices, the topological charge (4.1) can be represented in terms of matrices as

$$
\begin{aligned}
q^{(P)} & =\frac{-1}{32 \pi^{2}} N \sum_{\mu v \rho \sigma} \varepsilon_{\mu v \rho \sigma} \operatorname{tr}\left\{\hat{U}_{\mu}\left(\Gamma_{\mu} \hat{U}_{v} \Gamma_{\mu}^{\dagger}\right)\left(\Gamma_{v} \hat{U}_{\mu}^{\dagger} \Gamma_{v}^{\dagger}\right) \hat{U}_{v}^{\dagger}\right\}\left\{\hat{U}_{\rho}\left(\Gamma_{\rho} \hat{U}_{\sigma} \Gamma_{\rho}^{\dagger}\right)\left(\Gamma_{\sigma} \hat{U}_{\rho}^{\dagger} \Gamma_{\sigma}^{\dagger}\right) \hat{U}_{\sigma}^{\dagger}\right\} \\
& =\frac{-1}{32 \pi^{2}} N \sum_{\mu v \rho \sigma} \varepsilon_{\mu v \rho \sigma} \mathscr{Z}_{v \mu} \mathscr{Z}_{\rho \sigma} \operatorname{tr}\left(V_{\mu} V_{v} V_{\mu}^{\dagger} V_{v}^{\dagger} V_{\rho} V_{\sigma} V_{\rho}^{\dagger} V_{\sigma}^{\dagger}\right)
\end{aligned}
$$

Alternatively, one can rely on the so-called hybercube definition which leads to a star-product of matrices winding along the edges of the hybercube

$$
\begin{array}{r}
q^{(H)}=\frac{-1}{32 \pi^{2}} \sum_{x} \sum_{\mu \nu \rho \sigma} \varepsilon_{\mu v \rho \sigma} U_{\mu}(x) \star U_{v}(x+a \hat{\mu}) \star U_{\rho}(x+a \hat{\mu}+a \hat{v}) \star U_{\sigma}(x+a \hat{\mu}+a \hat{v}+a \hat{\rho}) \\
\left.\left.\left.\star U_{\mu}(x+a \hat{v}+a \hat{\rho}+a \hat{\sigma})^{\dagger}\right) \star U_{v}(x+a \hat{\rho}+a \hat{\sigma})^{\dagger}\right) \star U_{\rho}(x+a \hat{\sigma})^{\dagger} \star U_{\sigma}(x)^{\dagger}\right)(
\end{array}
$$

which reduces to the definition of the topological charge in $4 \mathrm{~d}$ gauge theory

$$
q^{(H)}=\frac{1}{32 \pi^{2}} \int d^{4} x \varepsilon_{\mu v \rho \sigma} G_{\mu v} \star G_{\rho \sigma}
$$

in the continuum limit.

By using the map between fields and matrices, the topological charge (4.1) can be represented in terms of matrices as

$$
\begin{array}{r}
q^{(H)=} \frac{-1}{32 \pi^{2}} N \sum_{\mu v \rho \sigma} \varepsilon_{\mu v \rho \sigma} \operatorname{tr}\left\{\hat{U}_{\mu}\left(\Gamma_{\mu} \hat{U}_{v} \Gamma_{\mu}^{\dagger}\right)\left(\Gamma_{v} \Gamma_{\mu} \hat{U}_{\rho} \Gamma_{\mu}^{\dagger} \Gamma_{v}^{\dagger}\right)\left(\Gamma_{\rho} \Gamma_{v} \Gamma_{\mu} \hat{U}_{\sigma} \Gamma_{\mu}^{\dagger} \Gamma_{v}^{\dagger}\right) \Gamma_{\rho}^{\dagger}\right) \\
\left.\left.\left(\Gamma_{\sigma} \Gamma_{\rho} \Gamma_{v} \hat{U}_{\mu}^{\dagger} \Gamma_{v}^{\dagger} \Gamma_{\rho}^{\dagger}\right) \Gamma_{\sigma}^{\dagger}\right)\left(\Gamma_{\sigma} \Gamma_{\rho} \hat{U}_{v}^{\dagger} \Gamma_{\rho}^{\dagger} \Gamma_{\sigma}^{\dagger}\right)\left(\Gamma_{\sigma} \hat{U}_{\rho}^{\dagger} \Gamma_{\sigma}^{\dagger}\right) \hat{U}_{\sigma}^{\dagger}\right\} \\
=\frac{-1}{32 \pi^{2}} N \sum_{\mu v \rho \sigma} \varepsilon_{\mu v \rho \sigma} \mathscr{Z}_{v \mu} \mathscr{Z}_{\rho v} \mathscr{Z}_{\sigma \rho} \mathscr{Z}_{\rho \mu} \mathscr{Z}_{\sigma v} \mathscr{Z}_{\sigma \mu} \operatorname{tr}\left(V_{\mu} V_{v} V_{\rho} V_{\sigma} V_{\mu}^{\dagger} V_{v}^{\dagger} V_{\rho}^{\dagger} V_{\sigma}^{\dagger}\right)
\end{array}
$$

The extension to higher dimensions is straight-forward. In practical studies, one can choose one or more planes noncommutative while leaving the others commutative [8].

\section{Conclusion and Outlook}

Today there exist several investigations of the topological sector of the two-dimensional noncommutative U(1) theory [3, 4]. Also classical solutions are available. The situation with the field-theoretic definition of instantons is reminiscent of lattice QCD where quantum gauge field configurations are topologically trivial and one needs to apply some smoothing procedure onto the gauge fields to unhide instantons.

In this contribution we worked out the field-theoretic definition to four and higher dimensions. We demonstrated that both the plaquette and hybercube definition can be taken over from the commutative gauge theory by respecting the star-multiplication and applying the map to the matrix model.

It would be interesting to adapt cooling techniques from QCD to the four-dimensional noncommutative $\mathrm{U}(1)$ theory [8]. At present we are working on this. It would be disirable to send the 
noncommutativity parameter $\theta$ of the four-dimensional noncommutative gauge theory to zero in order to obtain a realistic comparison of its topological content with the well-studied topological objects like instantons and monopoles in QCD.

Unfortunately, the transcription of a monopole observable seems to be difficult. The analogy to commutative $\mathrm{U}(1)$ theory of summing up the phases of the field over an elementary cube does not obviously transfer to the $\mathrm{U}(\mathrm{N})$ theory in the matrix model. Finding a reasonable definition one could be able to measure the monopole number on a noncommutative hypercube.

\section{References}

[1] R.J. Szabo, Quantum Field Theory on Noncommutative Spaces, Phys. Rept. 378 (2003) 207 [hep-th/0109162];

R.J. Szabo, Discrete Noncommutative Gauge Theory, Mod. Phys. Lett. A16 (2001) 367 [hep-th/0101216].

[2] W. Bietenholz, F. Hofheinz, J. Nishimura, Non-Commutative Field Theories beyond Perturbation Theory, Fortsch. Phys. 51 (2003) 745 [hep-th/ 0212258];

W. Bietenholz, F. Hofheinz, J. Nishimura, Y. Susaki, J. Volkholz, First Simulation Results for the Photon in a Non-Commutative Space, Nucl. Phys. Proc. Suppl. 140 (2005) 772

[hep-lat/0409059];

W. Bietenholz, A. Bigarini, F. Hofheinz, J. Nishimura, Y. Susaki, J. Volkholz, Numerical Results for U(1) Gauge Theory on $2 d$ and $4 d$ Non-Commutative Spaces, Fortsch. Phys. 53 (2005) 418 [hep-th/0501147].

[3] H. Aoki, J. Nishimura, Y. Susaki, The Index of the Overlap Dirac Operator on a Discretized $2 d$ Non-Commutative Torus, J. High Energy Phys. 02 (2007) 033 [hep-th/ 0602 078];

H. Aoki, J. Nishimura, Y. Susaki, Probability Distribution of the Index in Gauge Theory on $2 d$ Non-Commutative Geometry, J. High Energy Phys. 10 (2007) 024 [hep-th/ 0604093 ];

H. Aoki, J. Nishimura, Y. Susaki, Finite-Matrix Formulation of Gauge Theories on a Non-Commutative Torus with Twisted Boundary Conditions, J. High Energy Phys. 04 (2009) 055 [arXiv: 0810.5234 ];

H. Aoki, J. Nishimura, Y. Susaki, Dominance of a Single Topological Sector in Gauge Theory on Non-Commutative Geometry, J. High Energy Phys. 09 (2009) 084 [arXiv: 0907.2107 ].

[4] W. Frisch, H. Grosse, H. Markum, Instantons in Two-Dimensional Noncommutative U(1) Gauge Theory, POS (LATTICE 2007) 317;

R. Achleitner, W. Frisch, H. Grosse, H. Markum, F. Teischinger, Topology of Noncommutative U(1) Gauge Theory on the Lattice, Bled Workshops in Physics 8,1 (2007) 43.

[5] A. González-Arroyo, M. Okawa, A Twisted Model for Large-N Lattice Gauge Theory, Phys. Rev. D27 (1983) 2397.

[6] T. Eguchi, H. Kawai, Reduction of Dynamical Degrees of Freedom in the Large-N Gauge Theory, Phys. Rev. Lett. 48 (1982) 1063.

[7] L. Griguolo, D. Seminara, Classical Solutions of the TEK Model and Noncommutative Instantons in Two Dimensions, J. High Energy Phys. 03 (2004) 068 [hep-th/ 0311041$].$

[8] W. Bietenholz, J. Nishimura, Y. Susaki, J. Volkholz, A Non-Perturbative Study of 4d U(1) Non-Commutative Gauge Theory - the Fate of One-Loop Instability, J. High Energy Phys. 10 (2006) 042 [hep-th/0608072]. 